\title{
A Comparative Study of Single-Layer and Multi-Layer Traffic Engineering Approaches on Transparent Optical Networks
}

\author{
Namik Sengezer, Bart Puype*, Ezhan Karasan, Mario Pickavet* \\ Department of Electrical and Electronics Engineering, Bilkent University, Ankara, 06800, Turkey \\ Tel: 0090312 2901613, Fax:0090 312 2664192, e-mail: namik@ee.bilkent.edu.tr \\ *Department of Information Technology, Ghent University, Ghent, B-9000, Belgium
}

\begin{abstract}
This paper comparatively studies single-layer and multi-layer traffic engineering strategies on an IP/MPLS/WDM network. These strategies are evaluated and compared in two different scenarios. In the first scenario, the strategies make use of statistical information on the traffic patterns. In the second scenario, the traffic engineering decisions are based on the instantaneous traffic information only. The performance and benefits of both approaches are discussed based on simulations considering both throughput and network resource usage.
\end{abstract}

Keywords: traffic engineering, MPLS, WDM, logical topology configuration.

\section{INTRODUCTION}

Traffic engineering (TE) can improve the network performance by more efficient use of network resources when congestion in some part of the network degrades the performance. If TE is performed on the upper layer only, without changing the underlying logical topology, this traffic engineering method is called Single-Layer Traffic Engineering (STE). Another approach is to reconfigure the logical topology to optimize the network performance and resource utilization using the knowledge from the upper layer. This TE approach is called Multi-Layer Traffic Engineering (MTE) and it involves actions performed on both layers. Both approaches have their cons and pros; single layer traffic engineering's most important advantages being simplicity, less interaction among different layers and less signaling, while multi-layer traffic engineering provides better optimized topology, more efficient use of the network resources and better adaptation to the changing traffic patterns. There are a number of studies in the literature on single-layer [1]-[4] and multi-layer TE methods [5]-[9].

This study focuses on traffic engineering mechanisms on IP/MPLS networks with WDM technology in the underlying layer. Two previously proposed traffic engineering approaches are comparatively studied; the former is performed on a fixed WDM layer logical topology, and logical topology reconfigurations are involved in the latter method. Combining the most powerful aspects of these two TE approaches, a third TE strategy is also developed. The performances of these three TE strategies are evaluated on a common simulation platform under the same traffic demands. Two different cases are investigated: in the first case the TE strategies make use of available statistical information on the traffic demands, and in the second case there is no prior information. The benefits of both approaches and the effect of the prior traffic information are discussed based on the results.

The organization of the remainder of this paper is as follows. Section 2 describes the investigated traffic engineering strategies. In Section 3, simulation details are explained. The simulation results and their interpretation are discussed in Section 4, and Section 5 contains the conclusions.

\section{TRAFFIC ENGINEERING STRATEGIES}

Three different types of traffic engineering strategies are investigated in this study. The first strategy is suitable for the overlay model and is based on the optimization of the LSP routes on a fixed logical topology [10]. In the second approach, the WDM layer logical topology is reconfigured periodically to adapt to the changing traffic pattern [5]. The third strategy combines the advantages of both approaches, the logical topology is reconfigured periodically and between the topology updates, LSP routes are updated to better accommodate the LSP flows. The latter two strategies are applicable to overlay and augmented models.

\subsection{MPLS Layer TE on Fixed Logical Topology (TES-1)}

If some statistical information on the traffic demands is available, then using this information the network operator can design the WDM layer topology in the best way to optimize the network performance and the usage of the network resources. In this study, we focus on a 24 hour traffic pattern changing with the time of the day and assume that the statistical information is collected for every hour (The mechanisms of collecting the statistical data is out of the scope of this study). The available information is the maximum value of the traffic expectation between each source destination pair during each hour.

In this strategy, the first step is to design the WDM layer logical topology that supports the maximum amount of traffic from the given traffic matrix. This problem is referred as Multi-Hour Virtual Topology Design problem in the literature [11]. We utilize a heuristic algorithm utilizing tabu-search metaheuristic, to solve it. This algorithm is shown to have a satisfactory performance compared with lower bounds calculated by ILP 
solutions [10].

The virtual topology is designed considering the traffic expectation information. However, that kind of statistical traffic information is not exact in general and the actual traffic can significantly deviate from the expected value. To keep the blocking performance of the network at a certain level in such cases, a dynamic LSP rerouting algorithm is proposed. To this aim, a number of alternate shortest paths are calculated between the source and destination nodes of the LSP. To reroute an LSP, the best of these paths is chosen according to a cost function taking into account the link capacities and path lengths. The cost function is designed to choose the shorter paths when the network is lightly loaded and the paths with more residual capacity when the load on the lightpaths is high [10].

\subsection{Multi-layer TE with Periodic Logical Topology Updates (TES-2)}

In this strategy, both routing and topology generation are combined into a single step by relying on a load-based cost function for routing the MPLS flows [5]. The traffic is groomed onto fewer logical links than would be required when taking the direct-hop full-mesh approach, using appropriate link costs. This strategy considers the full-mesh topology in any case, in order to represent the high degree of flexibility in setting up lightpaths (logical links). Setting TE costs will allow some of these full-mesh links to remain unused by IP/MPLS traffic. Unused links will not be setup in the optical layer, so the actual logical topology is reduced.

The load-based link cost function has a high cost 'bump' for IP/MPLS links with a load below a certain threshold load, so the flows are groomed onto IP/MPLS links. It prevents ineffective use of the lightpath capacity by avoiding the setup of links for very small amounts of traffic. For the loads above a threshold, the cost function shows a steep increase and this prevents link overloads. Preventing overload is more important than grooming objectives, so costs for high loads always (need to) dominate. The resulting 'trench' in the cost function will concentrate link-loads in the optimal region and use the link capacities in the most efficient way.

\subsection{Multi-layer TE with Periodic Logical Topology Updates and Dynamic LSP Rerouting (TES-3)}

In this strategy, the WDM layer logical topology is reconfigured periodically as in the previous strategy. Between the topology update instances, a rerouting algorithm is utilized to optimize the routes of the LSP flows. The rerouting algorithm is the same as used in TES- 1 with the modification that the set of the shortest paths are recalculated after each logical topology reconfiguration. This approach combines the strong sides of the single layer and the multi-layer TE actions. While the WDM layer topology is reconfigured to cope with the large fluctuations in the traffic demands that occurs in a longer period, adaptation to the smaller changes are done with the rerouting actions in the upper layer.

\section{SIMULATIONS}

A common simulation platform is developed to evaluate the performances of the TE strategies. The traffic is modelled on the level of LSP flows. The traffic patterns representing the bandwidth expectations of traffic flows are generated by a method presented in [12]. A single LSP is established for each source destination pair and the changes in the traffic flow are modelled by updating the bandwidth requirement of the LSP. The bandwidth updates arrive according to a Poisson process and the update times belonging to each LSP are independent. While producing the actual traffic for simulations, to calculate the instantaneous bandwidth of an LSP, a zero mean Gaussian noise is added to the expected bandwidth value of that traffic flow at the bandwidth update instance. The standard deviation of the added noise is proportional to the expected bandwidth value. The ratio of the standard deviation to the expected value is denoted by the parameter $k$, which is used to represent the amount of uncertainty in the available traffic information [10].

Two different cases are investigated by simulations. In Case 1, for each traffic flow, the maximum value of the bandwidth expectation belonging to each hour is known. Each TE strategy uses this information when designing the logical topologies. In Case 2, the algorithms do not use any statistical information on the traffic, and the topology reconfigurations are performed using the instantaneous bandwidth requirements of the traffic flows.

\section{RESULTS}

Simulations are run for different values of the traffic uncertainty parameter k. For Case 1, the bandwidth blocking ratios for the three TE strategies are depicted in Figure 1. The number of lightpaths set up by each strategy is used as the metric representing usage of network resources. TES-2 and TES-3 established 20.5 lightpaths per hour on the average. To evaluate the performance of TES-1, two logical topologies are designed with 21 and 23 lightpaths.

As it is seen from the results, the strategies with topology updates, TES-2 and TES-3, outperform TES-1 which uses the fixed layer topology for the same number of utilized lightpaths. The logical topology reconfigurations require additional signalling and possible disruption of some traffic flows, however, these effects which are not investigated further in this study. Using more network resources, i.e., setting up more lightpaths, it is possible to achieve a similar performance to the dynamic topology TE strategies on a fixed 
logical topology. In the simulations TES-1 achieves this on a 23 lightpath network which corresponds to approximately $12 \%$ more resources.

An interesting observation is that, when the traffic unpredictability is low, TES-2 shows a better performance than TES-3 which performs LSP route updates between the topology reconfigurations. This is because if the traffic information used when designing the logical topologies is more precise, the routes calculated along with the topologies are better suited for the traffic patterns. When the unpredictability increases, rerouting the LSPs become effective in preventing blockings.

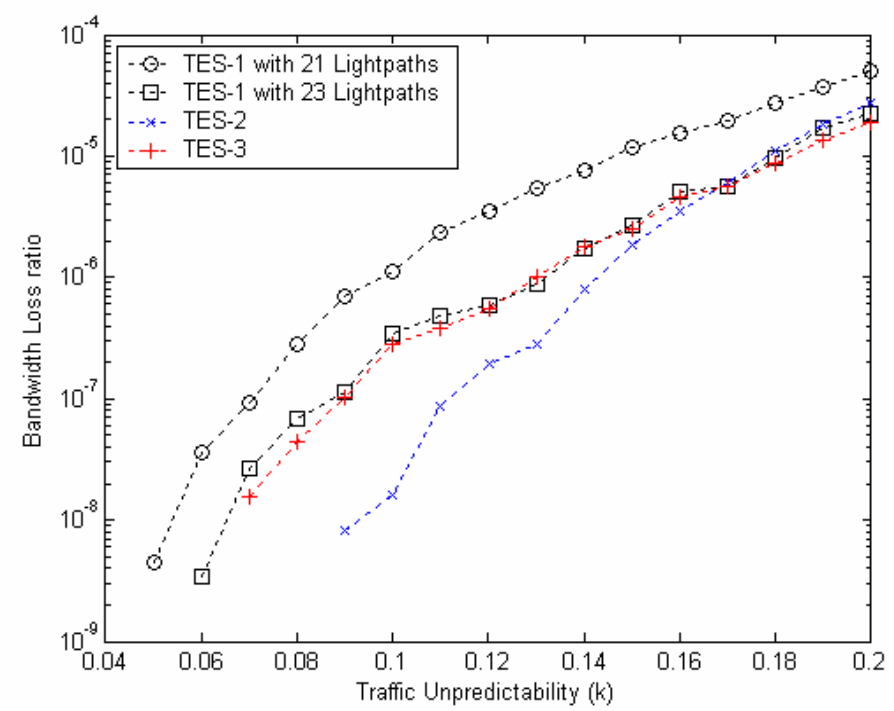

Figure 1. Bandwidth blocking ratios for Case 1.

The blocking performances of TES-2 and TES-3 are shown in Figure 2 for Case 2. Since TES-1 needs the statistical traffic information in order to design a fixed logical topology, it is not included in this case. The results show that, when there is no available traffic information, TES-3 has a better blocking performance than TES-2. This is expected since the previous results also showed that LSP rerouting improves the performance when the utilized traffic information is not accurate. Another important remark is the performance difference of the TE strategies for the two investigated cases. When the strategies make use of the information on the traffic expectation in Case 1, their performances improve several orders of magnitude. Even TES-1, which uses a fixed logical topology, outperforms the other two strategies when they use only the instantaneous traffic information.

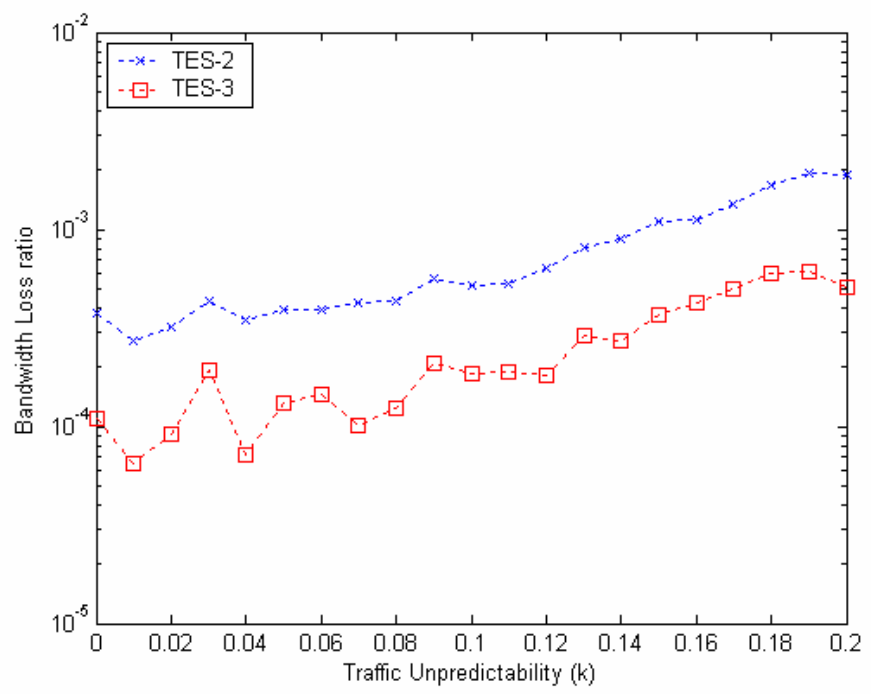

Figure 2. Bandwidth blocking ratios for Case 2. 


\section{CONCLUSIONS}

In this study, three TE strategies are comparatively studied. In the first strategy, the TE actions are carried out only in the upper (MPLS) layer. The other two TE strategies are multi-layer and perform also reconfigurations of the WDM layer logical topology. The simulation results show that logical topology updates have an important effect on the blocking performance and the efficient usage of network resources. In order to achieve a similar performance with only MPLS layer TE on a fixed logical topology, more network resources need to be utilized.

The effect of prior traffic information is also investigated by evaluating the performances of the TE strategies in two different cases. In the first case, the TE strategies make use of available information on the traffic expectation in each hour. In the second case, no prior information is available and the instantaneous traffic values are used performing TE. The results show that, if some statistical information on the traffic expectation is available, the TE performances can be improved by a considerable amount making use of this information when planning the TE actions. In the case of no or inaccurate prior information, between topology reconfiguration instances, the performance can be further improved with simple TE actions on the upper layer.

\section{ACKNOWLEDGEMENTS}

This work is supported in part by the IST-FP6 NoE project e-Photon/ONe+ and by the Scientific and Technological Research Council of Turkey (TUBITAK) under project EEEAG-104E047. Namik Sengezer is supported in part by the graduate scholarship of TUBITAK.

\section{REFERENCES}

[1] M. Kodialam, T. Lakshaman, Minimum Interface Routing With Applications to MPLS Traffic Engineering, in Proc. INFOCOM 2000, Tel Aviv, Israel, March 2000.

[2] M. Kodialam, T. Lakshaman, Dynamic routing of bandwidth guaranteed tunnels with restoration, in Proc. INFOCOM 2000, Tel Aviv, Israel, March 2000.

[3] S. Suri, M. Waldvogel, P. Warkhede, Profile Based Routing: A New Framework for MPLS Traffic Engineering, Quality of Future Internet Services, Lecture Notes in Computer Science 2156, 2001.

[4] B. Wang, X. Su, C. Chen: A New Bandwidth Guaranteed Routing Algorithm for MPLS Traffic Engineering, in Proc. ICC 2002, New York City, USA, May 2002.

[5] B. Puype, Q. Yan, D. Colle, S. De Maesschalck, I. Lievens, M. Pickavet, P. Demeester: Multi-layer traffic engineering in data-centric optical networks, in Proc. ONDM 2003, Budapest, Hungary, February 2003.

[6] J. Comellas, R. Martinez, J. Prat, V. Sales, G. Junyent: Integrated IP/WDM routing in GMPLS-based optical networks, Network, IEEE, vol. 17(2), pp. 22-7, 2003.

[7] E. Oki, K. Shiomoto, D. Shimazaki, N. Yamanaka, W. Imajuku, Y. Takigawa, Dynamic multilayer routing schemes in GMPLS-based IP+ optical networks, IEEE Communications Magazine, vol. 43(1), pp. 108-114, 2005.

[8] G. Retvari, P. Fodor, J. Tapolcai, T. Cinkler, Multi-layer traffic engineering schemes in GMPLS networks, in Proc. ICTON 2005, Barcelona, Spain, July 2005.

[9] P. Iovanna, R. Sabella, M. Settembre, A traffic engineering system for multilayer networks based on the GMPLS paradigm, Network, IEEE, vol. 17(2), pp. 28-37, 2003.

[10] N. Sengezer, E. Karasan, An Efficient Virtual Topology Design and Traffic Engineering Scheme for IP/WDM Networks, in Proc. ONDM 2007, Athens, Greece, July 2007 (to be published).

[11] M. Pioro, D. Medhi, Routing, Flow and Capacity Design in Communication and Computer Networks, Elsevier/Morgan Kaufmann, Amsterdam, 2004.

[12] J. Milbrandt, M. Menth, S. Kopf, Adaptive Bandwidth Allocation: Impact of Traffic Demand Models for Wide Area Networks, in Proc. ITC 19 (International Teletraffic Congress), Beijing, China, 2005. 